\title{
Circulating tumor cells in patients with metastatic castration resistant prostate cancer: exploratory findings at a tertiary referral hospital
}

This article was published in the following Dove Press journal:

Research and Reports in Urology

27 September 2014

Number of times this article has been viewed

\section{Sophie D Fosså \\ Siri L Hess' \\ Elisabeth Paus ${ }^{2}$ \\ Elin Borgen ${ }^{3}$}

'National Resource Center for Late Effects after Cancer Treatment, ${ }^{2}$ Department of Medical Biochemistry, ${ }^{3}$ Department of Pathology, Norwegian Radium Hospital, Oslo University Hospital, Radiumhospital, Oslo, Norway
Correspondence: Sophie D Fosså Oslo University Hospital, The Norwegian Radium Hospital, National Resource Center for Late Effects after Cancer Treatment, PO Box 4953 Nydalen, NO-0424 Oslo, Norway Email sdf@ous-hf.no
Objectives: In patients with metastatic castration-resistant prostate cancer (mCRPC), the finding of less than five circulating tumor cells (CTCs) $/ 7.5 \mathrm{~mL}$ blood before start of cytotoxic treatment or shortly thereafter indicates prolonged survival. In this descriptive pilot study, we investigated whether this association depends on the sequence of the therapeutic attempts.

Patients and methods: CTCs were determined in $41 \mathrm{mCRPC}$ patients before and 2-3 months after starting first-line treatment with docetaxel (group 1) or second-line treatment with either radium-223 (group 2) or placebo/best supportive care (group 3). A "favorable" CTC count was defined as $<5 \mathrm{CTC} / 7.5 \mathrm{~mL}$ blood. The results were related to overall survival.

Results: Pretreatment, six of ten men in group 1, three of 19 in group 2, and three of 12 patients in group 3 had a favorable CTC count, leading to a significant difference between first- and second-line therapy $(P=0.04)$. Decrease of pretreatment elevated CTCs to a favorable CTC count was significantly more often observed in patients on first-line therapy (three of four patients) than on second-line treatment (two of 26 men) $(P=0.03)$. A favorable CTC count before or shortly after treatment start was observed in nine of ten patients on first-line and in eight of 31 men on second-line therapy $(P=0.01)$. A favorable CTC count pretreatment or $2-3$ months after therapy start was associated with beneficial overall survival in the three groups combined and in each group analyzed separately.

Conclusion: In mCRPC, a favorable CTC count before or 2-3 months after start of therapy is associated with length of overall survival, though such favorable CTC counts are observed significantly less often in patients on second- than on first-line therapy.

Keywords: first-line treatment, second-line treatment, overall survival

\section{Introduction}

Metastatic castration-resistant prostate cancer (mCRPC) is a heterogeneous disease. The individual patient's condition is, though imperfectly, reflected by tumor markers, such as lactate dehydrogenase (LDH), prostate-specific antigen (PSA), and total alkaline phosphate (total ALP). Together with clinical parameters, such as pain ${ }^{1}$ and performance status, ${ }^{2-5}$ these biomarkers are included in prognostic models, which recently also have incorporated circulating tumor cells (CTCs). ${ }^{6-8}$ The finding of $<5 \mathrm{CTCs} / 7.5 \mathrm{~mL}$ blood before treatment start and/or a few weeks after treatment has been shown to be associated with favorable survival compared with patients with $\geq 5$ CTCs.

With the rapid development of new cytotoxic and hormonal therapies for $\mathrm{mCRPC}$, an increasing number of men will have received at least one life-prolonging systemic treatment before they are offered a new, often experimental therapy, frequently provided at a 
tertiary academic referral hospital. With this clinical scenario, one may question whether the prognostic significance of CTCs differs between patients in whom the new treatment represents a first-line or second-line treatment for mCRPC. ${ }^{9}$

The present analysis aimed to shed more light on this question by assessing the prognostic role of CTC counts determined pretreatment and during the early phase of the treatment, separately for first-line and second-line therapy of patients with mCRPC. We anticipated that the findings of $<5$ CTCs ("favorable" CTC count) would be associated with favorable survival in $\mathrm{mCRPC}$ patients receiving firstline therapy but not in those on second-line therapy, in whom favorable CTC counts would be observed less often than in those on first-line treatment.

\section{Patients and methods Patients}

Radium Hospital at the Oslo University Hospital serves as a tertiary referral hospital for mCRPC patients from the south-eastern health region in Norway. The hospital has participated in national and international multicenter studies concerning these patients. ${ }^{10-13}$ The Department of Pathology has broad experience with the determination of CTCs, ${ }^{14-16}$ using immunocytochemistry reverse-transcription polymerase chain reaction (RT-PCR) technology or the CellSearch ${ }^{\circledR}$ methodology.

After receiving written information, consenting patients with $\mathrm{mCRPC}$ were eligible for a prospective pilot study concerning determination of CTCs. All patients had progressed biochemically (PSA increase) or clinically (new metastases) during androgen deprivation treatment, and had a serum testosterone $<0.4 \mathrm{nmol} / \mathrm{L}$. None had received abiraterone or enzalutamide previously. The patients either were scheduled to start with docetaxel chemotherapy or, as part of the postdocetaxel option of the ALSYMPCA trial ${ }^{13}$ (patients in the placebo arm of the ALSYMPCA trial were eligible for the current study), received best supportive care (BSC), which could include prednisolone (10 mg daily per os) and/or palliative external beam radiotherapy. Thus three groups of patients were identified:

- Group 1, Docetaxel (75 mg/m² every third week) was applied as first-line treatment of mCRPC. None of these patients was subsequently included in either of the two other study groups

- Group 2, Radium-223_rec (Alpharadin) was given as second-line therapy, following docetaxel therapy performed at a local hospital before referral to Oslo University Hospital, Radium Hospital
- Group 3, Radium-223_plac: BSC was given, in the ALSYMPCA trial, as second-line therapy after previous docetaxel treatment at a local hospital.

\section{CTC determination}

In eligible patients, CTCs were determined no more than 1 week prior to start of the planned treatment (CTC_1). For all patients, a second CTC determination was planned after 2-3 months (CTC_2). For each CTC determination, blood was collected into CellSave Preservation Tubes (Janssen Diagnostics LLC), and 7.5 mL blood was analyzed per sample. Isolation and enumeration of CTCs were carried out using the Food and Drug Association (FDA)-approved CellSearch system (Janssen Diagnostics LLC), with cell interpretation following the standard CellSearch CTC definition. ${ }^{17}$ Following previous studies in mCRPC patients ${ }^{6,7}$ a finding of $<5$ CTCs/7.5 mL blood was defined as "favorable".

PSA, total ALP, bone-specific ALP (bone-ALP), and LDH were determined at the same days that the CTC determinations took place. LDH and total ALP were measured on a Cobas ${ }^{\circledR}$ L501 analyzer, while PSA was measured on Cobas L601 analyzer (F Hoffman-La Roche Ltd, Basel, Switzerland). Bone-ALP levels were determined with a MicroVue ${ }^{\text {TM }}$ BAP EIA kit (Quidel Corporation, San Diego, CA, USA).

\section{Statistics}

Using SPSS for Windows (Version 18), descriptive nonparametric tests were applied (median [range], for continuous variables). Differences between groups were assessed by the Fisher exact test or the Mann-Whitney test. Associations between pretreatment serum levels and CTC_1 counts were assessed by the Spearman's correlation test. Overall survival was determined by the Kaplan-Meier method, with the logrank test assessing differences between the estimates. The observation time started with the day of the first CTC determination and ended with the date of death or July 1, 2013, whichever occurred first. All tests were two-sided, setting the level of significance at $P<0.05$.

\section{Results}

\section{Patients}

Of 41 individual patients, 38 had been included into previously reported Phase III studies applying docetaxel ${ }^{12}$ or radium-223. ${ }^{13}$ All had multiple bone metastases, without visceral involvement. No significant difference emerged for pretreatment total ALP, bone-specific ALP or LDH comparing patients on first-line treatment with those on second-line therapy, while the median pretreatment PSA was lower in Group 1 than in the two other groups $(P=0.02)$. 
Table I Baseline characteristics

\begin{tabular}{|c|c|c|c|c|c|}
\hline \multirow[t]{3}{*}{$\mathbf{N}$} & \multirow{3}{*}{$\frac{\text { First-line treatment }}{\text { Group I }}$} & \multicolumn{3}{|c|}{ Second-line treatment } & \multirow[t]{3}{*}{ Total } \\
\hline & & \multirow{2}{*}{$\frac{\text { Group } 2}{\text { Radium-223_rec }}$} & \multirow{2}{*}{$\frac{\text { Group } 3}{\text { Radium-223_plac }^{\text {b }}}$} & \multirow[t]{2}{*}{ Subtotal } & \\
\hline & & & & & \\
\hline Patients & 10 & 19 & 12 & 31 & 41 \\
\hline Age (years) & $68(63-75)^{c}$ & $69(52-83)$ & $68(63-86)$ & $70(52-86)$ & $69(52-86)$ \\
\hline PSA $(\mu g / L)$ & $49(2-I, 520)$ & $352(12-3,740)$ & $425(4-4,850)$ & $388(4-4,850)$ & $293(2-4,850)$ \\
\hline \multicolumn{6}{|l|}{ LDH (U/L) } \\
\hline \multirow[t]{2}{*}{ Elevated $^{d}$} & $233(169-651)$ & $215(177-760)$ & 357 (199-750) & $291(177-760)$ & $290(169-760)$ \\
\hline & 4 & 8 & II & 19 & 23 \\
\hline \multicolumn{6}{|l|}{ Total ALP (U/L) } \\
\hline \multirow[t]{2}{*}{ Elevated } & $137(63-3,268)$ & $208(48-829)$ & I89 (48-758) & 218 (48-829) & $180(48-3,268)$ \\
\hline & 8 & 15 & 9 & 24 & 32 \\
\hline \multicolumn{6}{|l|}{ Bone-ALP (U/L) } \\
\hline \multirow[t]{2}{*}{ Elevated } & $46(27-2,039)$ & $99(17-562)$ & $83(10-538)$ & $99(10-562)$ & $80(10-2,039)$ \\
\hline & 6 & 15 & 9 & 24 & 30 \\
\hline Observation time (months) & $29(I-42)$ & II (3-37) & $13(2-23)$ & II (I-36) & $12(1-42)$ \\
\hline \multicolumn{6}{|l|}{ Vital status } \\
\hline Alive & 3 & 3 & 0 & 3 & 6 \\
\hline Dead & 7 & 16 & 12 & 28 & 35 \\
\hline
\end{tabular}

Notes: aRadium-223 received; ${ }^{b}$ placebo/best supportive care; ' $m e d i a n$ (range); ${ }^{\text {nnumber }}$ of patients with values above the upper limit of the normal range.

Abbreviations: ALP, total alkaline phosphate; Bone-ALP, bone-specific ALP; LDH, lactate dehydrogenase; PSA, prostate-specific antigen.

\section{CTC counts}

Ten patients with mCRPC had CTCs determined before their first-line docetaxel treatment, and for 31 men, CTC_1 counts were available before their second-line treatment (Table 2). CTC_2 counts were performed in 34 men after median 2.8 months (range: 1.8-3.9).

Median CTC counts, expressed as CTC/7.5 mL blood, differed nonsignificantly between patients from group 1 and those from group 2 and group 3 combined (2 [0-141] vs 18 $[0-1,524])(P=0.16)$ (Table 2). Six of the ten men on first-line therapy had CTCs $<5$ before treatment start compared with six of 31 men receiving second-line treatment $(P=0.04)$. In three of four paired samples from group1 elevated CTC_1 counts have decreased to $<5$ at the second determination, the comparable figures in group 2 and group 3 combined being two of 23 men $(P=0.03)$. In none of the eight evaluable patients with elevated CTC_1 in the placebo arm was a favorable CTC count observed at the second CTC determination. Overall, favorable CTC counts before or shortly after treatment start were observed in nine of ten men in group 1 (first-line treatment) and in eight of 31 patients in group 2 and 3 combined (second-line therapy) $(P=0.01)$. No significant associations were observed between the individual CTC_1 counts and pretreatment PSA, total ALP, bone-ALP, or LDH (data not shown).

\section{Survival}

Patients from group 1 lived significantly longer than those from group 2 or group $3(P=0.037)$ (Figure 1A). Combining

Table 2 Findings related to CTC counts pretreatment or 2-3 months after treatment start

\begin{tabular}{|c|c|c|c|c|c|}
\hline \multirow[t]{3}{*}{$\mathbf{N}$} & \multirow{3}{*}{$\begin{array}{l}\text { First-line treatment } \\
\text { Group I } \\
\text { Docetaxel }\end{array}$} & \multicolumn{3}{|c|}{ Second-line treatment } & \multirow[t]{3}{*}{ Total } \\
\hline & & \multirow{2}{*}{$\begin{array}{l}\text { Group } 2 \\
\text { Radium-223_rec }^{\mathrm{a}}\end{array}$} & \multirow{2}{*}{$\frac{\text { Group 3 }}{\text { Radium-223_plac }^{\mathrm{b}}}$} & \multirow[t]{2}{*}{ Subtotal } & \\
\hline & & & & & \\
\hline Patients & 10 & 19 & 12 & 31 & 41 \\
\hline \multicolumn{6}{|l|}{ Pretreatment } \\
\hline \multicolumn{6}{|l|}{ CTC_I } \\
\hline Median (range) & $2(0-|4|)$ & $15(0-756)$ & $28(0-1,524)$ & $18(0-1,524)$ & $15(0-1,524)$ \\
\hline Patients with $<5$ & $6 / 10(60 \%)$ & $3 / 19(16 \%)$ & $3 / 12(25 \%)$ & $6 / 31(19 \%)$ & $12 / 4(29 \%)$ \\
\hline \multicolumn{6}{|l|}{ 2-3 months follow up } \\
\hline $\begin{array}{l}\text { Patients with CTC_I } \geq 5 \text { decreasing } \\
\text { to } C T C \_2<5\end{array}$ & $3 / 4(75 \%)$ & $2 / 15$ & $0 / 8$ & $2 / 23(9 \%)$ & $5 / 27(19 \%)$ \\
\hline CTC_I $<5$ or CTC_ $2<5$ (all patients) & $9 / 10$ (91\%) & $5 / 19$ & $3 / 12$ & $8 / 31$ (26\%) & $|7 / 4|(4 \mid \%)$ \\
\hline
\end{tabular}

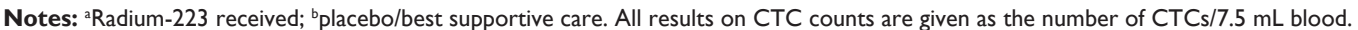
Abbreviations: CTC, circulating tumor cells; CTC_I, pretreatment CTC count; CTC_2, CTC count at 2-3 months posttreatment. 


\section{A}

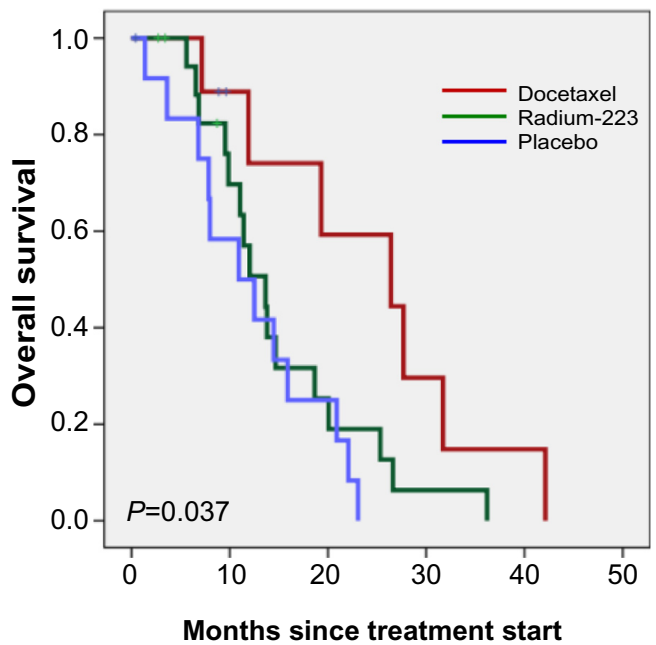

B

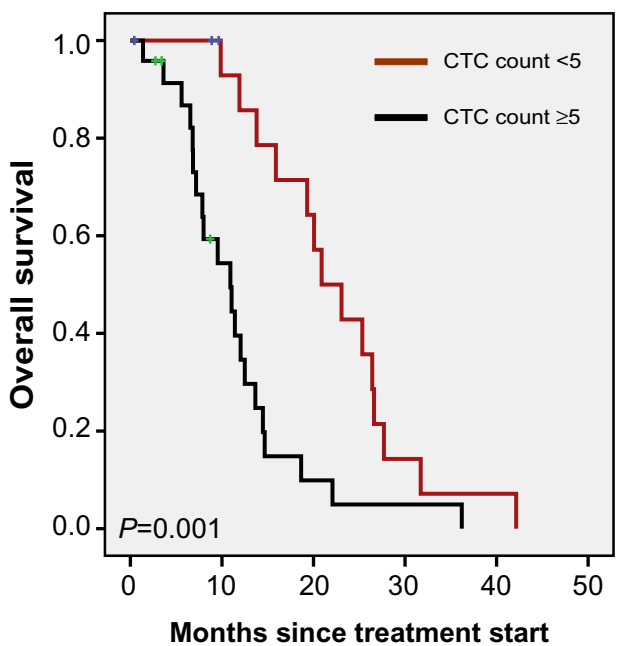

Figure I Overall survival and CTC counts per $7.5 \mathrm{~mL}$ blood in 41 patients with $\mathrm{mCRPC}$.

Notes: (A) First-line vs second-line treatment: ten patients were on docetaxel as first-line treatment; 19 patients received radium-223 as second-line treatment; and 12 patients received placebo/best supportive care as second-line treatment. (B) CTC-counts before treatment start and after 2-3 months; all treatment groups.

Abbreviations: CTC, circulating tumor cells; mCRPC, metastatic castration-resistant prostate cancer.

all three groups, a favorable CTC count before treatment start or 2-3 months thereafter was associated with significantly improved overall survival $(P=0.001)$ (Figure 1B). These latter results were supported by survival analyses performed separately in each of the three groups; however, the numbers of patients in the different subgroups (with or without favorable CTC counts) were very small (Figure $2 \mathrm{~A}-\mathrm{C}$ ), the median survival time being 27, 19, and 20 months (Kaplan-Meier estimates), respectively, for group 1, group 2 , and group 3.

\section{Discussion}

This admittedly small sample is, as far as we know, the first to point to differences related to the presence of favorable CTC counts and their prognostic information when the sequence of therapeutic attempts is considered: The finding of $<5 \mathrm{CTCs} / 7.5$ $\mathrm{mL}$ blood before or shortly after the treatment initiation was significantly more often observed in patients on first-line than on second-line treatment. The observation of $<5 \mathrm{CTCs} / 7.5 \mathrm{~mL}$ blood before start of treatment or 2-3 months posttreatment indicated a favorable prognosis, both among patients on first-
A

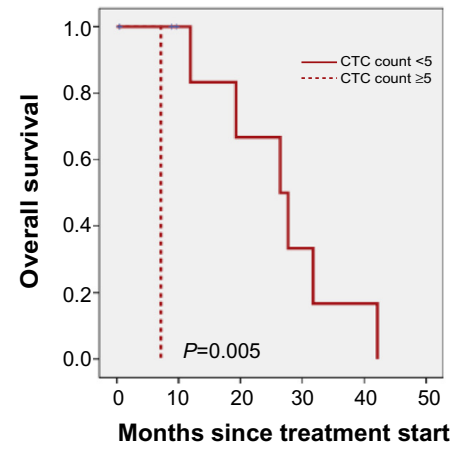

B

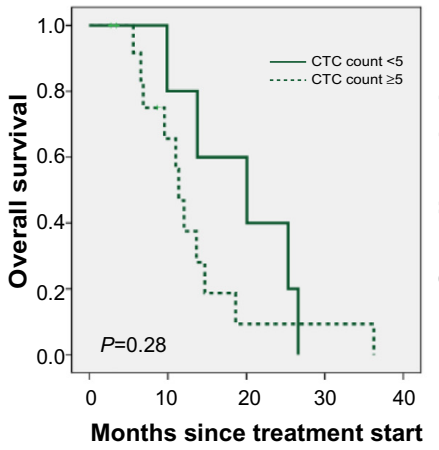

C

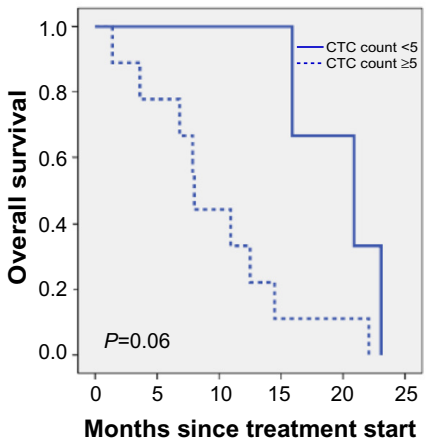

Figure 2 CTC counts per $7.5 \mathrm{~mL}$ blood pretreatment or after 2-3 months and overall survival in each of the three groups; (A) Group I (first-line docetaxel); group 2 (second-line ra-223); and (C) group 3 (second-line placebo).

Notes: Different scaling is used in the $\mathrm{x}$-axis. (A) In patients receiving first-line docetaxel, nine patients had CTC count $<5$ and one patient had CTC count $\geq 5$; (B) in patients receiving second-line ra-223, five patients had CTC count $<5$ and I 4 patients had CTC count $\geq 5$; and (C) in patients receiving second-line placebo (best supportive care), three patients had CTC count $<5$ and nine patients had CTC count $\geq 5$.

Abbreviation: CTC, circulating tumor cells. 
line treatment and in those on second-line therapy. However, the length of overall survival associated with a favorable CTC count was reduced after second-line therapy compared with first-line treatment.

When starting cytotoxic treatment in MCRPC, in particular in scientific trials, today's clinicians are at an increasing degree challenged to estimate future life expectancy in the individual patient. In clinical trials that evaluate new anticancer regimens in $\mathrm{mCRPC}$ patients, overall survival is an important endpoint, requiring reliable prognostic criteria to adjust for the heterogeneity of the patients. Routinely available biomarkers, such as PSA, hemoglobin, total ALP, LDH, and results from new imaging techniques serve only limitedly as valid objective prognostic parameters, even if combined in multivariate models. The presence of severe pain and/or of a reduced performance status is recognized as a subjectively assessed prognostic factor. ${ }^{5,9}$ In this situation, clinicians need more objective biomarkers associated with survival. Indeed, investigators have recently become aware of the prognostic significance of the number of an mCRPC patient's previous therapeutic attempts. Reanalyzing data from a previously randomized trial, Halabi et al have thus published a prognostic nomogram restricted to patients with second-line therapy. ${ }^{5}$ CTC counts may represent new independent prognostic factors in such models.

However, differences as to prognostic information emerging from CTC determinations have to be expected, related to the number of preceding therapeutic attempts: Compared with men on first-line treatment, favorable CTC counts were in our pilot study, significantly less often observed in patients on second-line therapy. Nevertheless, the prognostic role of these CTC findings was maintained if the patients on first- and second-line treatment are analyzed separately, though with differing survival duration. We were surprised that significant survival differences emerged in a cohort of only 41 patients, even when our three groups were analyzed separately. Whether one should use categorized CTC counts, ${ }^{6}$ probably preferred by clinicians, or present CTC results as continuous variables ${ }^{7}$ remains debatable. The fact that none of the patients of the placebo arm (group 3) experienced reduction of CTCs indicates sufficient robustness of our technique.

Due to small numbers within each group, no survival analyses were made according to the decrease of pretreatment elevated CTCs, as done by Olmos et $\mathrm{al}^{6}$ and Scher et $\mathrm{al}^{7}{ }^{7}$ though significant differences were observed in the paired samples as to the event of decrease to a favorable CTC count.
Our findings support clinical experience regarding survival of $\mathrm{mCRPC}$ patients related to the number of the individual patient's previous treatment courses. Only the most recent trials of drugs have considered this effect, as for example the abiraterone trials. ${ }^{18,19}$ In these trials, the progression-free survival of the chemotherapy-naïve patients exceeded that of patients who received abiraterone after docetaxel by approximately 10 months. A comparable though smaller difference of progression-free survival was observed in patients in the placebo arm of the ALSYMPCA trial, ${ }^{13}$ when docetaxel-naïve patients are analyzed separately from those after docetaxel failure ( $\sim 8$ months before docetaxel vs $\sim 3$ months postdocetaxel). These observations again indicate that the number of previous therapeutic attempts represent prognostic factors requiring new nomograms. ${ }^{5,9}$ In the future, an increasing number of mCRPC patients will receive third- and fourthline treatment, emphasizing the need for taking into account the number of preceding therapeutic attempts in addition to "traditional" prognostic factors (PSA, total ALP, LDH, pain, performance status). The identification of reliable prognostic factors is particularly important for clinicians at tertiary referral hospitals as their patients with $\mathrm{mCRPC}$ will have received several new hormonal or cytotoxic therapies prescribed by private specialists or uro-oncologists at local hospitals with oncological units before they finally are referred to a tertiary academic uro-oncological unit for inclusion in trials testing experimental drugs.

Our survival data are generally below those documented by Olmos et al, ${ }^{6}$ probably due to the fact that our hospital acts as a tertiary referral hospital. More than $80 \%$ of the patients, especially those in group 2 and group 3, used opiates due to highly advanced mCRPC with multiple bone metastases on bone scan.

Apart for the small size of our cohort, several limitations of our entirely descriptive study must be considered. Though the life expectancy was estimated to be $>6$ months in all of our patients at start of treatment, the vast majority of them had advanced bone metastases, as commonly seen in patients referred to a tertiary referral hospital. Therefore, findings on CTCs in relation to the number of therapeutic attempts may differ at institutions seeing earlier cases of mCRPC. Due to the small number of patients, the risk of type 2 errors must not be overlooked. Therefore, no multivariate analysis was performed. Finally the inclusion of patients on placebo/ BCS only may be debatable. However, we consider BCS as a relevant second-line management in many patients with mCRPC and have therefore included these patients in our analyses. 
We consider it a strength that we differentiated our findings between first-line and second-line treatment, though we are aware of the need for larger patient cohorts to facilitate multivariate analyses including treatment line as a covariate.

In conclusion, CTC determination performed before or shortly after initiation of treatment among patients with mCRPC provides clinically worthwhile prognostic information. Favorable CTC counts ( $<5$ CTCs/7.5 mL blood) are significantly more often observed in men on first-line than on second-line treatment, the former findings associated with significantly longer survival than those observed in men with second-line therapy. The final significance of a favorable CTC count before or early during treatment of mCRPC has to be determined by multivariate analyses taking into account the number of preceding therapeutic attempts together with traditional prognostic factors.

\section{Disclosure}

The authors report no conflicts of interest in this work.

\section{References}

1. Halabi S, Vogelzang NJ, Kornblith AB, et al. Pain predicts overall survival in men with metastatic castration-refractory prostate cancer. J Clin Oncol. 2008;26(15):2544-2549.

2. Halabi S, Small EJ, Kantoff PW, et al. Prognostic model for predicting survival in men with hormone-refractory metastatic prostate cancer. J Clin Oncol. 2003;21(7):1232-1237.

3. Fosså SD, Dearnaley DP, Law M, Gad J, Newling DW, Tveter K. Prognostic factors in hormone-resistant progressing cancer of the prostate. Ann Oncol. 1992;3(5):361-366.

4. Smaletz O, Scher HI, Small EJ, et al. Nomogram for overall survival of patients with progressive metastatic prostate cancer after castration. J Clin Oncol. 2002;20(19):3972-3982.

5. Halabi S, Lin CY, Small EJ, et al. Prognostic model predicting metastatic castration-resistant prostate cancer survival in men treated with secondline chemotherapy. J Natl Cancer Inst. 2013;105(22):1729-1737.

6. Olmos D, Arkenau HT, Ang JE, et al. Circulating tumour cell (CTC) counts as intermediate end points in castration-resistant prostate cancer (CRPC): a single-centre experience. Ann Oncol. 2009;20(1):27-33.
7. Scher HI, Jia X, de Bono JS, et al. Circulating tumour cells as prognostic markers in progressive, castration-resistant prostate cancer: a reanalysis of IMMC38 trial data. Lancet Oncol. 2009;10(3):233-239.

8. Goldkorn A, Ely B, Quinn DI et al. Circulating tumor cell counts are prognostic of overall survival in SWOG S0421: a phase III trial of docetaxel with or without atrasentan for metastatic castration-resistant prostate cancer. J Clin Oncol. 2014;32:1136-1142.

9. Omlin A, Pezaro C, Mukherji D, et al. Improved survival in a cohort of trial participants with metastatic castration-resistant prostate cancer demonstrates the need for updated prognostic nomograms. Eur Urol. 2013;64(2):300-306.

10. Fosså SD, Slee PH, Brausi M, et al. Flutamide versus prednisone in patients with prostate cancer symptomatically progressing after androgen-ablative therapy: a phase III study of the European organization for research and treatment of cancer genitourinary group. $J$ Clin Oncol. 2001;19(1):62-71.

11. Fosså SD, Jacobsen AB, Ginman C, et al. Weekly docetaxel and prednisolone versus prednisolone alone in androgen-independent prostate cancer: a randomized phase II study. Eur Urol. 2007;52(6): 1691-1698.

12. Meulenbeld HJ, van Werkhoven ED, Coenen JL, et al. Randomised phase II/III study of docetaxel with or without risedronate in patients with metastatic Castration Resistant Prostate Cancer (CRPC), the Netherlands Prostate Study (NePro). Eur J Cancer. 2012;48(16):2993-3000.

13. Parker C, Nilsson S, Heinrich D, et al; ALSYMPCA Investigators. Alpha emitter radium-223 and survival in metastatic prostate cancer. N Engl J Med. 2013;369(3):213-223.

14. Molloy TJ, Bosma AJ, Baumbusch LO, et al. The prognostic significance of tumour cell detection in the peripheral blood versus the bone marrow in 733 early-stage breast cancer patients. Breast Cancer Res. 2011;13(3):R61.

15. Mathiesen RR, Borgen E, Renolen A, et al. Persistence of disseminated tumor cells after neoadjuvant treatment for locally advanced breast cancer predicts poor survival. Breast Cancer Res. 2012;14(4):R117.

16. Seeberg LT, Waage A, Brunborg C, et al. Circulating Tumor Cells in Patients With Colorectal Liver Metastasis Predict Impaired Survival. Ann Surg. Epub February 6, 2014.

17. Allard WJ, Matera J, Miller MC, et al. Tumor cells circulate in the peripheral blood of all major carcinomas but not in healthy subjects or patients with nonmalignant diseases. Clin Cancer Res. 2004;10(20): 6897-6904.

18. de Bono JS, Logothetis CJ, Molina A, et al; COU-AA-301 Investigators. Abiraterone and increased survival in metastatic prostate cancer. $N$ Engl J Med. 2011;364:1995-2005.

19. Ryan CJ, Smith MR, de Bono JS, et al; COU-AA-302 Investigators. Abiraterone in metastatic prostate cancer without previous chemotherapy. N Engl J Med. 2013;368(2):138-148.
Research and Reports in Urology

\section{Publish your work in this journal}

Research and Reports in Urology is an international, peer-reviewed, open access journal publishing original research, reports, editorials, reviews and commentaries on all aspects of adult and pediatric urology in the clinic and laboratory including the following topics: Pathology, pathophysiology of urological disease; Investigation and treatment of

\section{Dovepress}

urological disease; Pharmacology of drugs used for the treatment of urological disease. The manuscript management system is completely online and includes a very quick and fair peer-review system, which is all easy to use. Visit http://www.dovepress.com/testimonials.php to read real quotes from published authors. 